\title{
Reducing the Emittance Growth during Transition Crossing via the rf Manipulation and the $\gamma_{\mathrm{T}}$ Jump
}

\author{
Xi Yang, Charles M. Ankenbrandt, and Valeri A. Lebedev \\ Fermi National Accelerator Laboratory \\ Box 500, Batavia IL 60510
}

\begin{abstract}
Usually there is a large longitudinal emittance growth during transition crossing in Booster. The combination of both the rf manipulation and the $\gamma_{\mathrm{T}}$ jump can reduce the longitudinal emittance growth during transition to a negligible level. So the goal for Booster to be able to deliver $8-\mathrm{GeV}$ proton beams to Main Injector at the intensity of $4.5 \times 10^{12}$ per batch with $\varepsilon_{L}$ of $0.12 \mathrm{eV} \cdot \mathrm{sec}$ and $\Delta \mathrm{p}$ of $18 \mathrm{MeV}$ can be achieved.
\end{abstract}

\section{Introduction}

The maximum beam intensity in Main Injector is limited by the longitudinal emittance $\left(\varepsilon_{L}\right)$ and momentum spread $(\Delta p)$ of the $8-\mathrm{GeV}$ beam before it's transferred from Booster to Main Injector.[1-3] Usually there is a large $\varepsilon_{L}$ growth during transition crossing (TC), so it is important for us to reduce the $\varepsilon_{L}$ growth via the rf manipulation, the transition jump, or the combination of both.[4,5]

Since the longitudinal space charge (SC) forces defocus the beam bunch before transition and focus the beam after transition, and also, the synchronous motions are frozen during $\mathrm{TC}$, manipulating the beam in the longitudinal phase space via the rf voltage can be implemented.[4] Besides, the existing transition-jump $\left(\gamma_{\mathrm{T}}\right)$ system (TJS) 
can be used to reduce the deleterious effects of a high intensity beam passing through transition via reducing the time that the beam spends near the transition energy.[5,6] One expects that the combination of both the rf manipulation and the $\gamma_{\mathrm{T}}$ jump would provide a further reduction in the $\varepsilon_{L}$ growth of the beam during TC, and numerical simulations are used to find the answer for this.

\section{Numerical Investigations}

ESME simulations are used to search the optimal setting for the combination of both the rf manipulation and the $\gamma_{\mathrm{T}}$ jump during TC.

Since the RFSUM curve including the rf manipulation during TC already has been optimized for the purpose of minimizing $\varepsilon_{L}$ and $\Delta p$ of the 8-GeV proton beam,[4] it can be directly used in ESME simulations. Except that the timing of these two rf manipulating pulses, one right before transition and one right after transition, should be adjusted relative to the new transition gate time when the TJS is used. The time relationship between each one of the rf manipulating pulses and the transition gate is fixed no matter what $\gamma_{\mathrm{T}}$ jump value is used, since the rf manipulation is particularly optimized to reduce the $\varepsilon_{L}$ growth of the beam during TC.[4] Different transition gates should be set for different $\gamma_{\mathrm{T}}$ jump values according to the Booster $\gamma$ ramp, as shown in Fig. 1(a),[7] and the result is shown in Fig. 1(b). Five different $\gamma_{\mathrm{T}}$ jump values $\left(\Delta \gamma_{\mathrm{T}}\right)$ are used in ESME simulations, and they are 0.0, 0.3, 0.4, 0.5, and 0.6. Measured $\gamma_{T}$ waveforms at maximum $\gamma_{\mathrm{T}}$ jump values of $0.3,0.4,0.5$, and 0.6 , are shown as the black, red, green, and blue curves in Fig. 1(c) respectively, and they are used in ESME simulations.

ESME simulations are done for two different extracted beam intensities of $4.0 \times 10^{12}$ and $4.5 \times 10^{12}$ protons, at the configuration of combining both the $\mathrm{rf}$ manipulation and the $\gamma_{\mathrm{T}}$ jump during TC. Five different $\gamma_{\mathrm{T}}$ jump values, 0.0, 0.3, 0.4, 0.5, and 0.6 , are simulated. The longitudinal emittance $\left(\varepsilon_{L}\right)$ with $95 \%$ of the beam at $8 \mathrm{GeV}$ vs. the $\gamma_{\mathrm{T}}$ jump $\left(\Delta \gamma_{\mathrm{T}}\right)$ is shown in Fig. 2(a), $\Delta p$ in rms at the $8-\mathrm{GeV} v s . \Delta \gamma_{\mathrm{T}}$ is shown in Fig. 2(b), and the black and red curves in Figs. 2(a) and (b) correspond to extracted beam intensities of $4.0 \times 10^{12}$ and $4.5 \times 10^{12}$ protons respectively. Without the rf manipulation at 
TC and at the extracted beam intensity of $4.0 \times 10^{12}$ protons, $\varepsilon_{L}$ with $95 \%$ of the beam and $\Delta p$ in rms at $8-\mathrm{GeV} v s . \Delta \gamma_{\mathrm{T}}$ are shown as the green curve in Figs. 2(a) and (b).

As shown in Fig. 2(a), at the extracted beam intensity of $4.0 \times 10^{12}$ protons, the minimum $\varepsilon_{L}$ at $8 \mathrm{GeV}$ is achieved at the configuration of combining both the $\mathrm{rf}$ manipulation and the $\gamma_{\mathrm{T}}$ jump of 0.6 during TC, and it is $0.052 \mathrm{eV} \cdot \mathrm{sec}$. There is a $15.3 \%$ $(\approx(0.0614-0.052) / 0.0614))$ reduction in $\varepsilon_{L}$ and a $\left.8.7 \%(\approx(3.156-2.88) / 3.156)\right)$ reduction in $\Delta p$ compared to the situation with the $\gamma_{\mathrm{T}}$ jump only (at the same value of $\Delta \gamma_{\mathrm{T}}$ $=0.6)$; there is a $14.8 \%(\approx(0.061-0.052) / 0.061)$ reduction in $\varepsilon_{L}$ and a $3.5 \%$ $(\approx(2.985-2.88) / 2.985))$ reduction in $\Delta p$ compared to the situation with the $\mathrm{rf}$ manipulation only; and there is a $41 \%(\approx(0.088-0.052) / 0.088)$ reduction in $\varepsilon_{L}$ and a $20 \%(\approx(3.58-2.88) / 3.58))$ reduction in $\Delta p$ compared to the situation without both the rf manipulation and the $\gamma_{\mathrm{T}}$ jump.

\section{Conclusions}

Usually there is a large $\varepsilon_{L}$ growth during TC at the normal operation (without both the rf manipulation and the $\gamma_{T}$ jump). For a Gaussian distribution, $\varepsilon_{L}$ is six time EPSILON. At the extracted beam intensity of $4.0 \times 10^{12}$ protons, EPSILON vs. time is shown in Fig. 3(a), and $\varepsilon_{L}$ is nearly doubled after TC. However, the combination of both the rf manipulation and the $\gamma_{\mathrm{T}}$ jump during TC can reduce the $\varepsilon_{L}$ growth to about $4 \%$ $(\approx(0.052-0.05) / 0.052)$, as shown in Fig. 3(b). Comparing situations with and without both the rf manipulation and the $\gamma_{\mathrm{T}}$ jump, there is a $41 \%$ reduction in $\varepsilon_{L}$ and a $20 \%$ reduction in $\Delta p$.

With the combination of both the rf manipulation and the $\gamma_{T}$ jump, at the extracted beam intensity of $4.0 \times 10^{12}$ protons, $\varepsilon_{L}$ of $0.052 \mathrm{eV} \cdot \mathrm{sec}$ with $95 \%$ of the beam and $\Delta p$ in

rms of $2.88 \mathrm{MeV}$ at $8-\mathrm{GeV}$ can be achieved; at the extracted beam intensity of $4.5 \times 10^{12}$ protons, $\varepsilon_{L}$ of $0.055 \mathrm{eV} \cdot \mathrm{sec}$ and $\Delta p$ of $2.92 \mathrm{MeV}$ at $8-\mathrm{GeV}$ can also be achieved. There is only a $6 \%$ increase in $\varepsilon_{L}$ from the extracted beam intensity of $4.0 \times 10^{12}$ protons to $4.5 \times 10^{12}$ protons, provide that the TJS setting should be readjusted for each beam intensity. 


\section{References:}

[1] K. Koba, etc., "SLIP STACKING EXPERIMENTS AT FERMILAB MAIN INJECTOR”, FERMILAB-CONF-03-107.

[2] K. Koba and J. Steimel, "SLIP STACKING”, FERMILAB-CONF-02-205.

[3] X. Yang, etc., "Reducing the Momentum Spread of the 8-GeV proton Beam via the Bunch Rotation in Booster", FERMILAB-FN-0769-AD, submitted.

[4] X. Yang, etc., "Reducing the Longitudinal Emittance of the 8-GeV Beam via the rf Manipulation in a Booster Cycle”, FERMILAB-FN-0769-AD, submitted.

[5] W. Merz, etc., “Transition Jump System for the Fermilab Booster”, FERMILAB-TM1473.

[6] X. Yang, etc., "Study Report of the Booster Transition Jump System”, FERMILABTM-2287-AD.

[7] X. Yang, etc., "Booster 6-GeV Study”, FERMILAB-TM-2283-AD. 


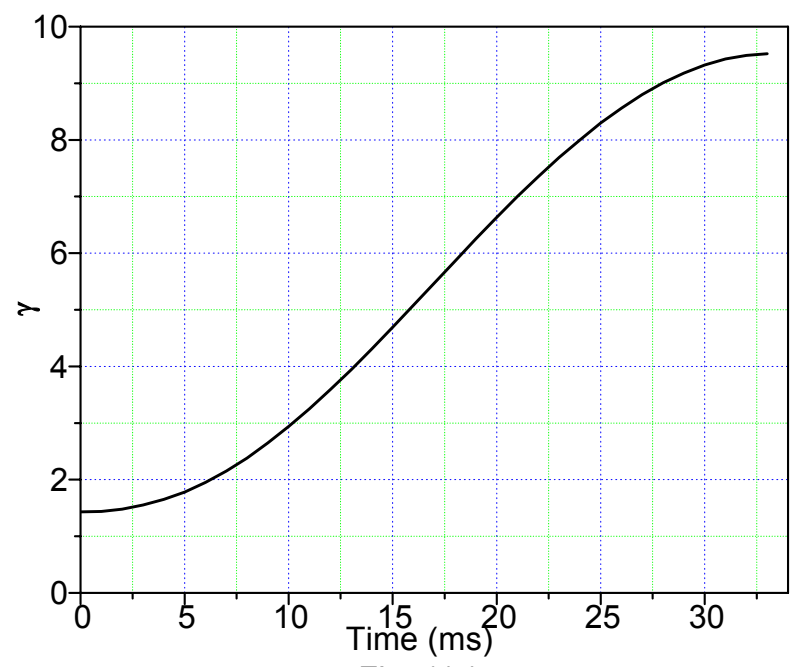

Fig. 1(a)

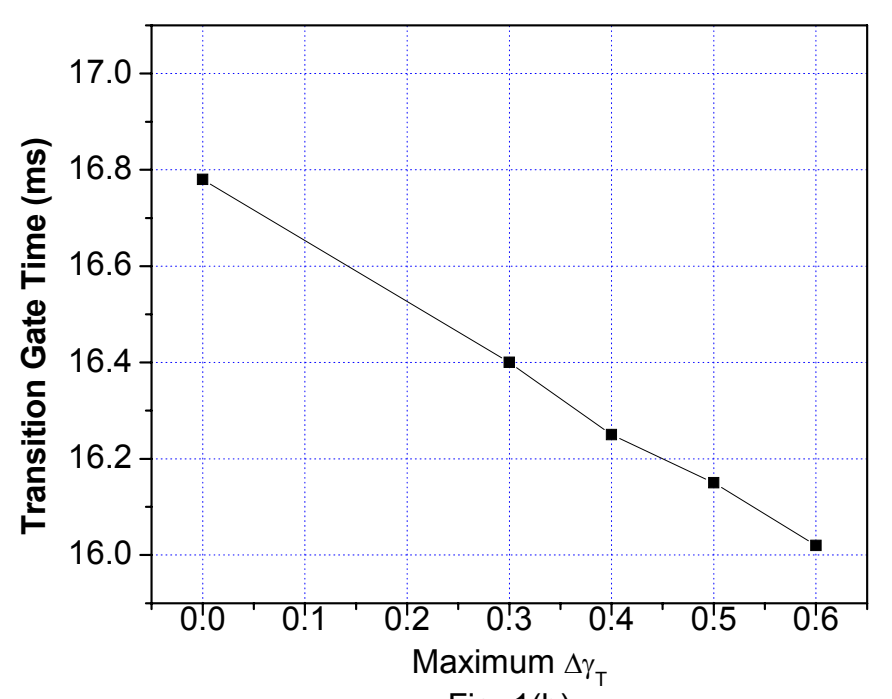

Fig. 1(b) 


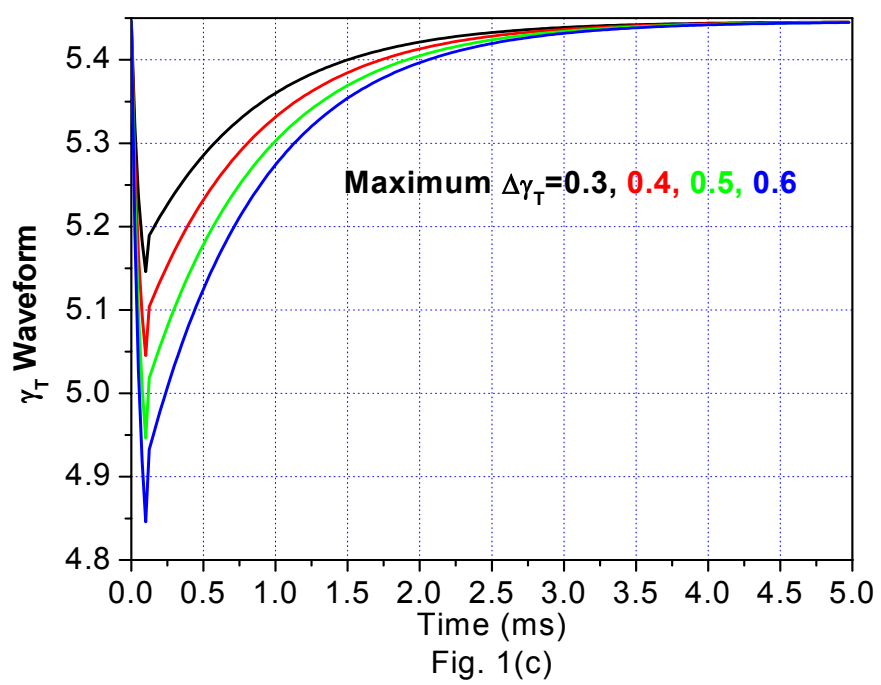

Fig. 1(a) the Lorentz relativistic factor $(\gamma) v s$. the time in a Booster cycle.

Fig. 1(b) the transition gate time $v s$. the $\gamma_{\mathrm{T}}$ jump value $\left(\Delta \gamma_{\mathrm{T}}\right)$.

Fig. 1(c) $\gamma_{T}$ waveforms at maximum $\gamma_{T}$ jump values of $0.3,0.4,0.5$, and 0.6 , are shown as the black, red, green, and blue curves respectively. 


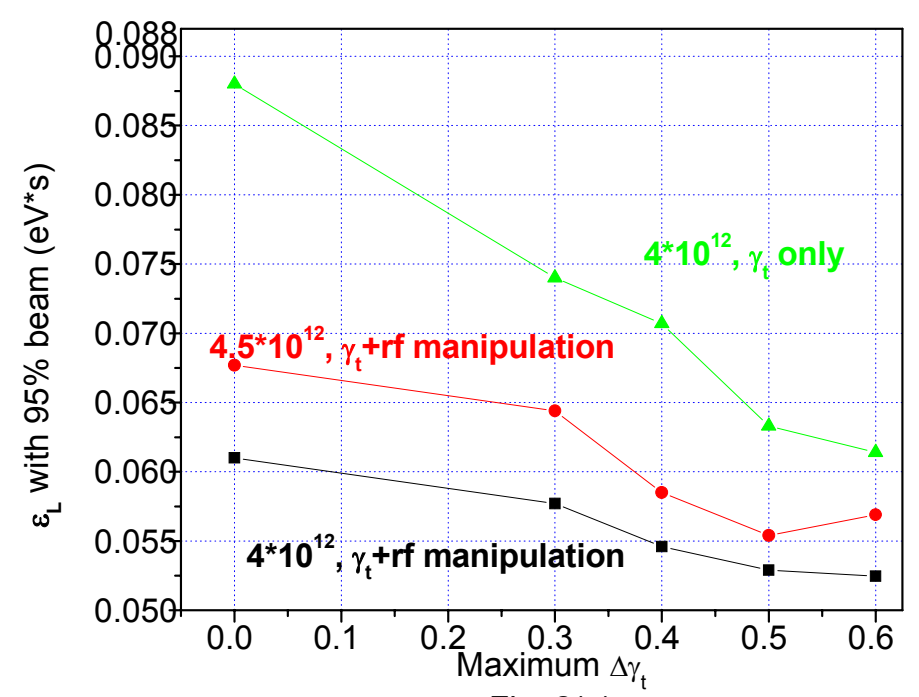

Fig. 2(a)

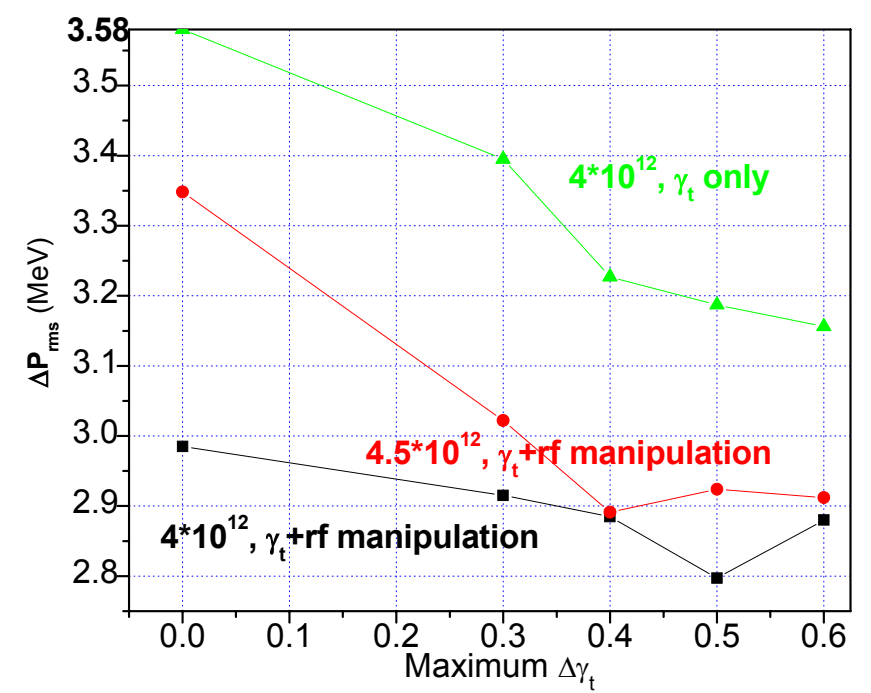

Fig. 2(b)

The black and red curves in Figs. 2(a) and (b) correspond to extracted beam intensities of $4.0 \times 10^{12}$ and $4.5 \times 10^{12}$ protons respectively at the configuration of combining both the $\mathrm{rf}$ manipulation and the $\gamma_{\mathrm{T}}$ jump during TC, and the green curve in Figs. 2(a) and (b) is at the extracted beam intensity of $4.0 \times 10^{12}$ protons and without the $\mathrm{rf}$ manipulation at TC.

Fig. 2(a) $\boldsymbol{\varepsilon}_{L}$ with $95 \%$ of the beam at $8 \mathrm{GeV} v s . \Delta \gamma_{\mathrm{T}}$.

Fig. 2(b) $\Delta p$ in rms at the $8-\mathrm{GeV}$ vs. $\Delta \gamma_{\mathrm{T}}$. 


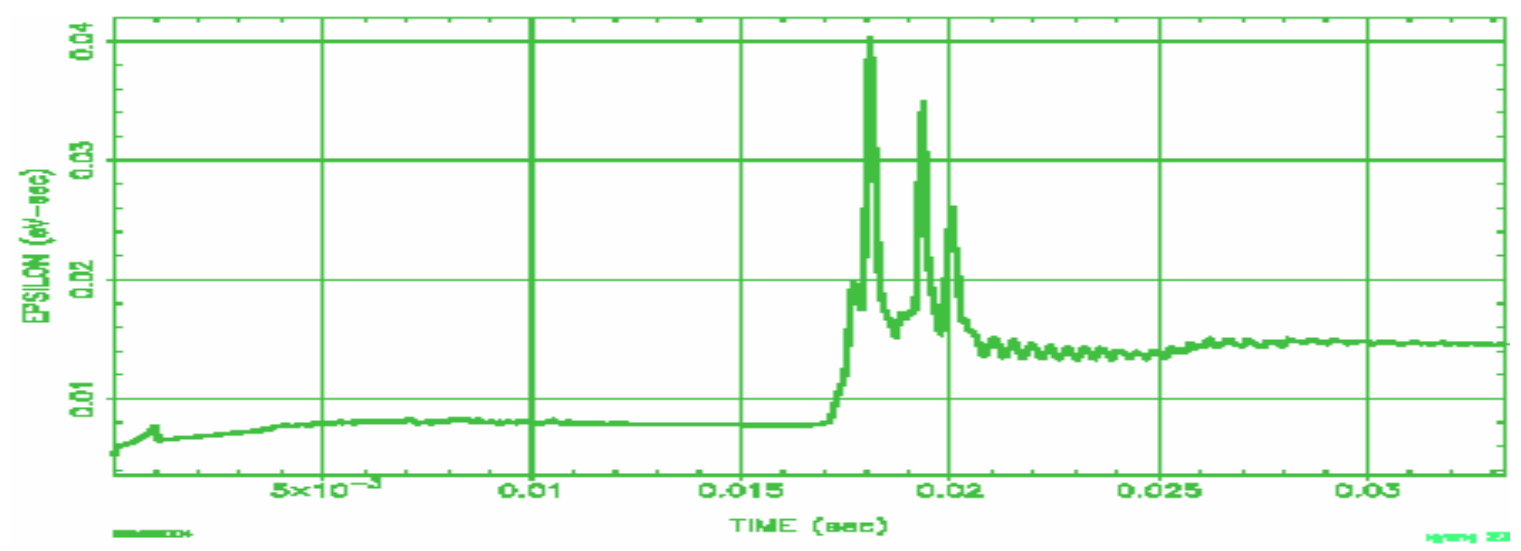

Fig. 3(a)

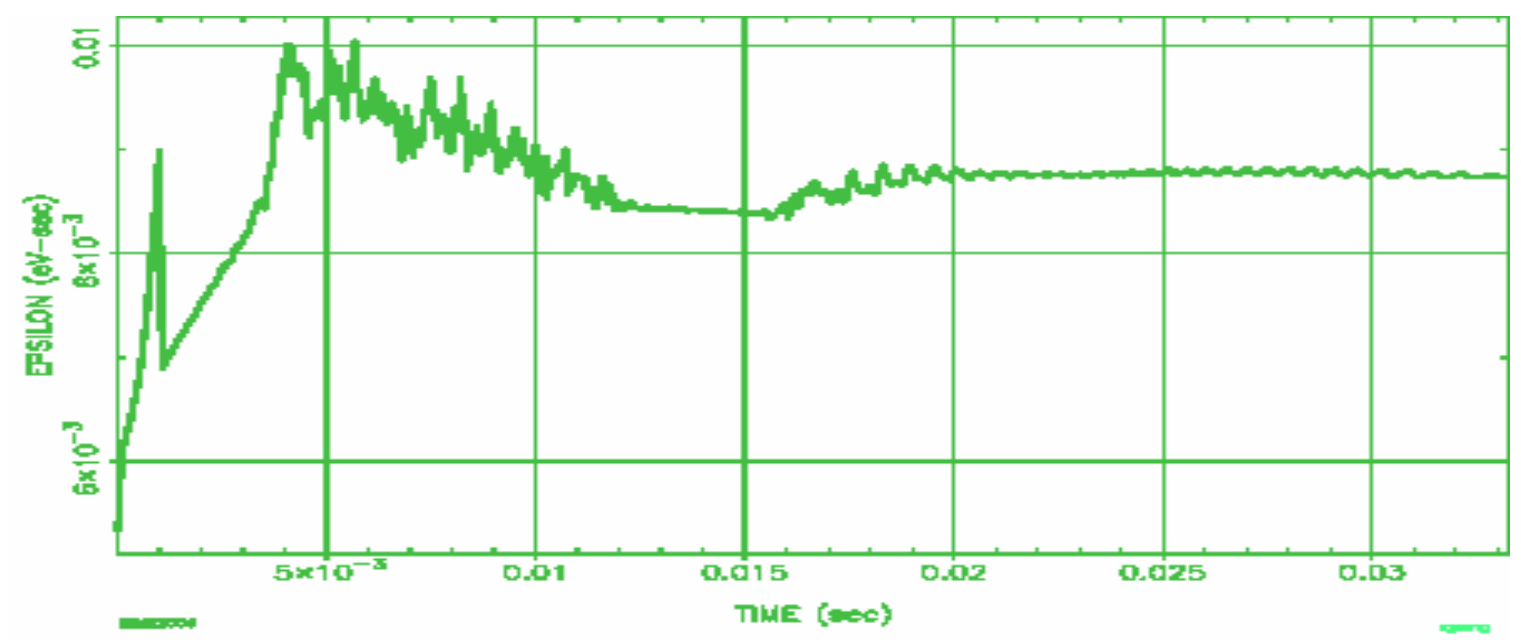

Fig. 3(b)

Fig. 3(a) without both the rf manipulation and the $\gamma_{\mathrm{T}}$ jump, at the extracted beam intensity of $4.0 \times 10^{12}$ protons, EPSILON vs. time.

Fig. 3(b) with the combination of both the rf manipulation and the $\gamma_{\mathrm{T}}$ jump during TC, at the extracted beam intensity of $4.0 \times 10^{12}$ protons, EPSILON $v s$. time. 